\title{
Manejo de tejidos periimplantarios con provisionales atornillados en Agenesia bilateral: Reporte de caso
}

\author{
Management of peri-implant tissues with screwed provisionals in bilateral agenesis: Case report
}

\author{
Raquel Guerra ${ }^{1,2, a}$,Anthony Bolaños ${ }^{2,3, a}$, Pablo Chávez ${ }^{2,4, b}$
}

\section{RESUMEN}

Una de las alternativas de tratamiento para los casos de agenesia de incisivos laterales en el sector superior es mediante la rehabilitación con implantes dentales. Es importante realizar un adecuado diagnóstico y plan de tratamiento respetando los tiempos biológicos para la regeneración ósea y manejo de tejidos, de tal manera el resultado final cumpla con la estética que involucra el caso. El objetivo del presente trabajo es reportar un caso clínico de un paciente de sexo masculino de 24 años de edad con agenesia bilateral superior de los incisivos laterales, recibió tratamiento de ortodoncia por 6 años, para la fase definitiva del tratamiento rehabilitador involucró la regeneración ósea horizontal de las zonas afectadas con injerto autólogo de mentón, la planificación de la colocación de los implantes y el manejo de tejidos periimplantarios.

PALABRAS CLAVE: Anodoncia, implantes dentales, contorno gingival.

\begin{abstract}
One of the treatment alternatives for cases of lateral incisors agenesis in the upper zone is through rehabilitation with dental implants. It is important to make an adequate diagnosis and a treatment plan, respecting biological times for bone regeneration and tissue management, so that the final result complies with the aesthetics that the case involves. The objetive of the present work is to report a clinical case of 24 year old male patient with superior bilateral agenesis of the lateral incisors, previously receiving orthodontic treatment for 6 years, the next part of the treatment involved horizontal bone regeneration of the affected areas with autologous chin grafting, the planning of implant placement and the management of periimplant tissues.
\end{abstract}

KEY WORDS: Anodontia, dental implants, gingival contour. 


\section{INTRODUCCIÓN}

La agenesia es un desorden congénito de naturaleza hereditaria, sin embargo, los trastornos localizados pueden explicar la agenesia dental como una probable actividad mutagénica. Existen reportes de prevalencia de agenesia en dentición permanente en un rango de $2,2 \%$ a $7,6 \%$ siendo más propensas la población femenina, la mayoría de los casos reportados presentan la ausencia de uno o dos dientes, dentro de esta ausencia se destaca la agenesia bilateral particularmente de los incisivos laterales superiores $(1,2)$. Una de las alternativas de tratamiento es la colocación de implantes intraóseos y una rehabilitación con coronas sobre estos. Para obtener un resultado aceptable en el sector anterior es ideal la posición tridimensional del implante y el manejo de los tejidos blandos con provisionales (3). La modificación del perfil de emergencia en el contorno crítico y subcrítico son esenciales para optimizar la arquitectura del tejido periimplantario, en el caso de implantes inmediatos el contorno crítico determina la ubicación del margen gingival mientras que el contorno subcrítico modifica el volumen del tejido blando mediante una configuración cóncava o convexa, siendo esta última la que ofrece mayor volumen en el tejido blando, estos contornos son áreas dinámicas que pueden modificarse durante el acondicionamiento del tejido maduro en casos tardíos (4). El objetivo del reporte de caso clínico es describir la secuencia clínica del manejo de tejidos blandos en un paciente con agenesia bilateral rehabilitado con implantes dentales que involucre la alta estética. La justificación es presentar una secuencia en el manejo de tejidos blandos con provisionales en casos de agenesia bilateral que se sustenta en la necesidad de un adecuado diagnóstico y planificación en la colocación de implantes para lograr una armonía en la arquitectura gingival y que las restauraciones se integren imperceptiblemente con los dientes adyacentes.

\section{Presentación del caso}

Paciente de sexo masculino de 24 años de edad, de raza mestiza, sin antecedentes sistémicos y oclusalmente estable, que fue transferido al servicio de Rehabilitación Oral del Posgrado y Especialización de la Universidad Peruana Cayetano Heredia, Lima, Perú. Recibió previamente tratamiento ortodóncico en el servicio de Ortodoncia y Ortopedia Maxilar por 6 años. Al examen intraoral presentó agenesia de las piezas 12 y 22 con evidencia de defectos óseos, presenta fenotipo periodontal grueso (figura 1). Al examen radiográfico se observa disminución generalizada de las raíces (Rizomicri), presenta ensanchamiento del ligamento periodontal generalizado (figura 2). Para el plan de trabajo para el diagnóstico se obtuvieron modelos de estudio primario, realizando el encerado para la planificación del tratamiento del sector anterosuperior. Al análisis tomográfico se evidenció poca disponibilidad ósea, motivo por el cual se planificó dos injertos en bloque de mentón con hueso particulado y plasma rico en plaquetas previo a la colocación de implantes dentales (figura 3 y figura 4). Pasado el tiempo de osteointegración (6 meses) se colocaron dos implantes Strong WS Cono Morse (SIN ${ }^{2}$, Sao Paulo, Brasil) de $3.5 \mathrm{~mm} \times 11 \mathrm{~mm}$. En la segunda fase, se confeccionaron provisionales de acrilico termocurado Vitalloy ${ }^{\circledR}$ (Vitacryl; A. Tarrillo

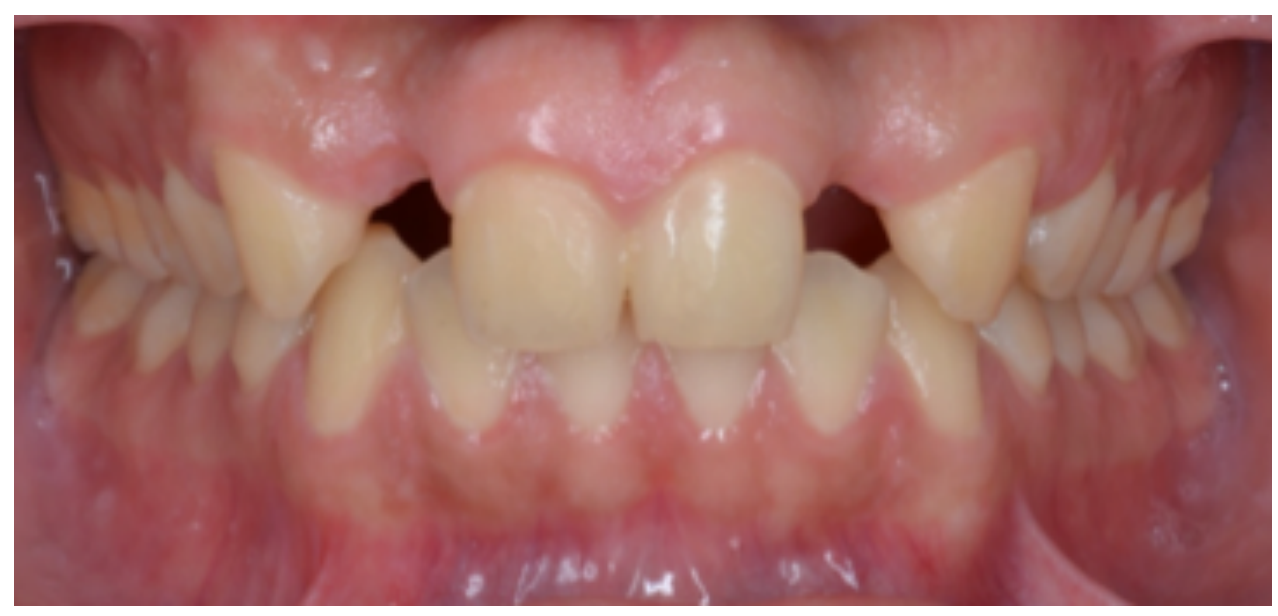

Figura 1. Examen intraoral presentó agenesia de las piezas 12 y 22 . 


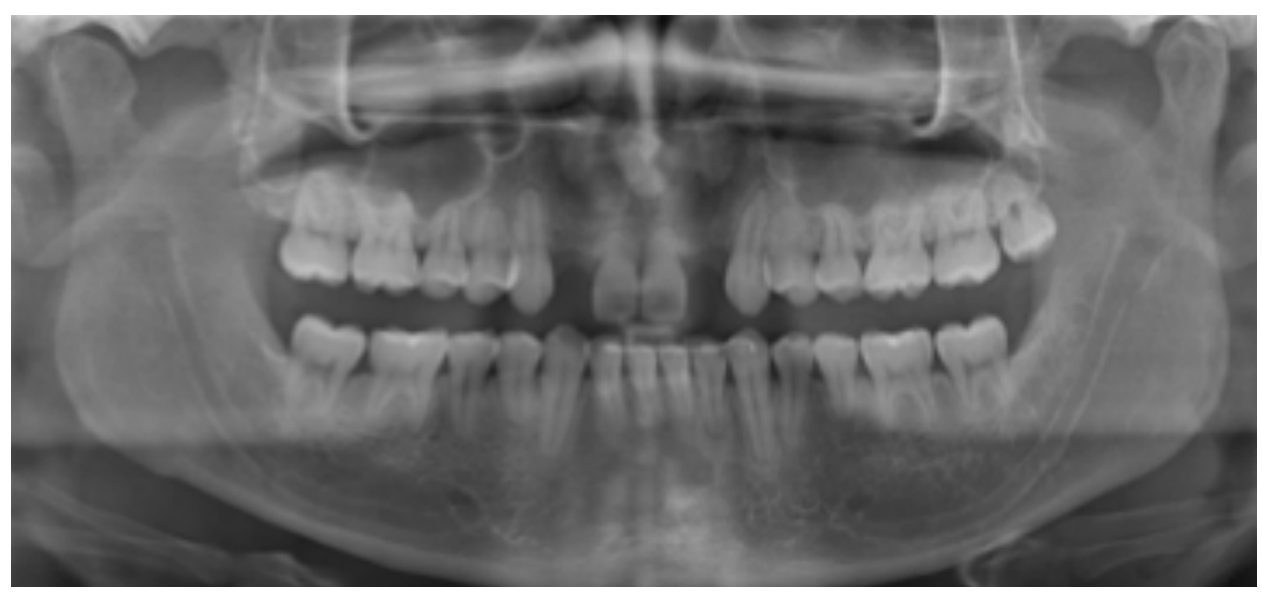

Figura 2. Examen radiográfico
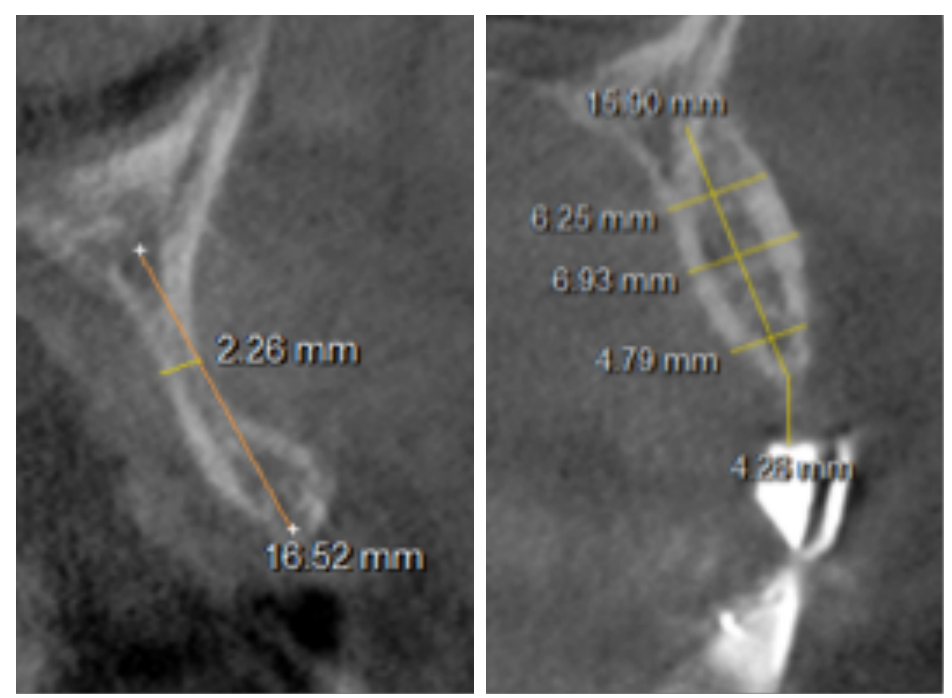

Figura 3. Análisis tomográfico.
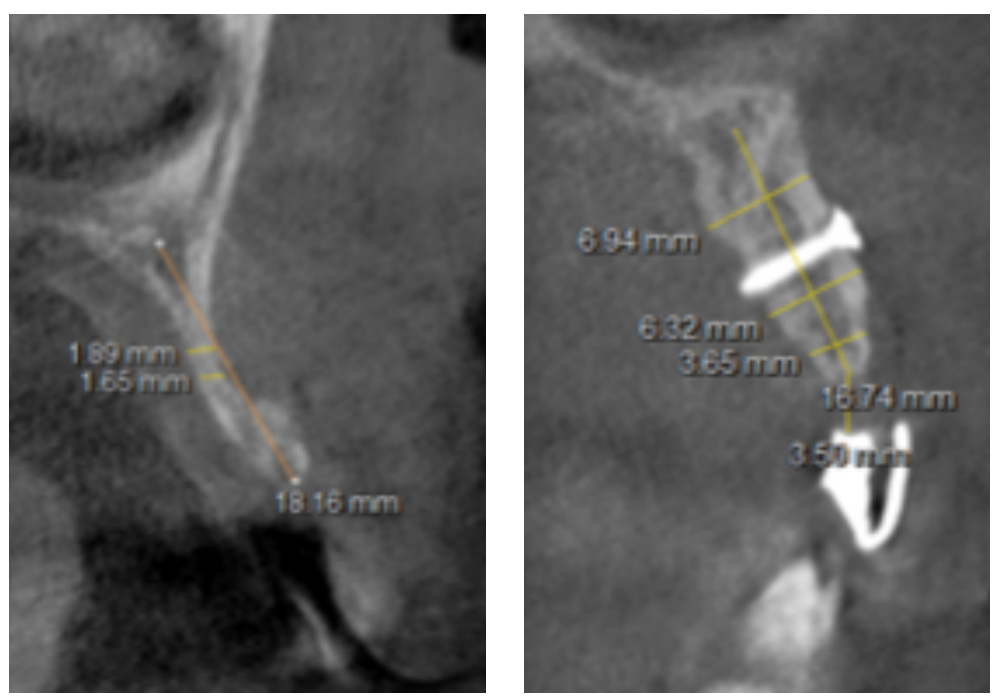

Figura 4. Análisis tomográfico. 
Barba S.A., Lima, Perú) para el manejo de los tejidos blandos a nivel de las piezas 12 y 22, los cuales estuvieron fijados a un cilindro provisional de titanio $(\mathrm{SIN} \AA$, Sao Paulo, Brasil) atornillado al implante, verificando la adaptación de este al implante mediante una radiografía periapical (figura 5). El cilindro fue recortado dando una altura de $6 \mathrm{~mm}$, los provisionales fueron agujerados en el medio (figura 6), de tal forma que el cilindro calce en el medio del espacio edéntulo, se cubrió la entrada del cilindro con teflón (Golfo USA ${ }^{\circledR}$ ) y se capturó el cilindro con acrílico de autocurado Duralay color 62 (Reliance Dental Manufacturing LLC, Illinois USA) (figura 7). Después del acrilizado se retiró el provisional, en la parte cervical se realizó un incrementó con resina fluida (Filtek ${ }^{\mathrm{TM}}$ Z350XT Flow - 3M ESPE Dental Products. St. Paul, Minnesota, USA) formando el contorno crítico a $2 \mathrm{~mm}$ por debajo del zenit de los incisivos centrales de tal manera que entre los zenits de los incisivos centrales, laterales y caninos formen una zona triangular. El contorno subcrítico se realizó de forma convexa (figura 7 y figura 8). Se finalizó con el acabado y pulido del provisional con gomas de grano grueso (gris) y grano fino (verde) (Politip, Ivoclar-Vivadent; Schaan, Liechtenstein). Se manejó la conformación de los tejidos blandos en 2 citas mediante la adición por capas de resina compuesta (Filtek $^{\mathrm{TM}}$ Z350 - 3M ESPE Dental Products. St. Paul, Minnesota, USA) a nivel del contorno crítico y subcrítico a las 2 semanas y 4 semanas de la instalación del provisional (figura 9 y figura 10). Se logró conformar las papilas interdentales, el contorno crítico y subcrítico observando que los tejidos se encontraran estables, sin inflamación, con el color de la encía igual a la encía adyacente; todo esto nos indicó que se podía realizar la impresión definitiva. La instalación final se realizó con coronas metal cerámicas cementadas (figura 11 y figura 12).

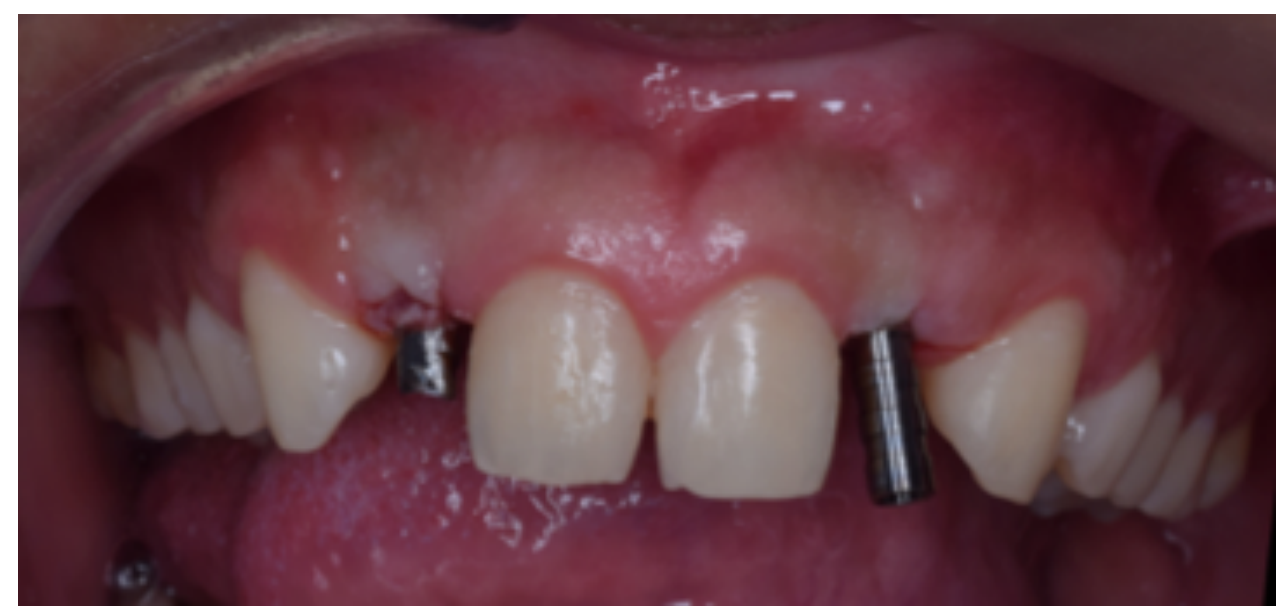

Figura 5. Implante de provisionales.

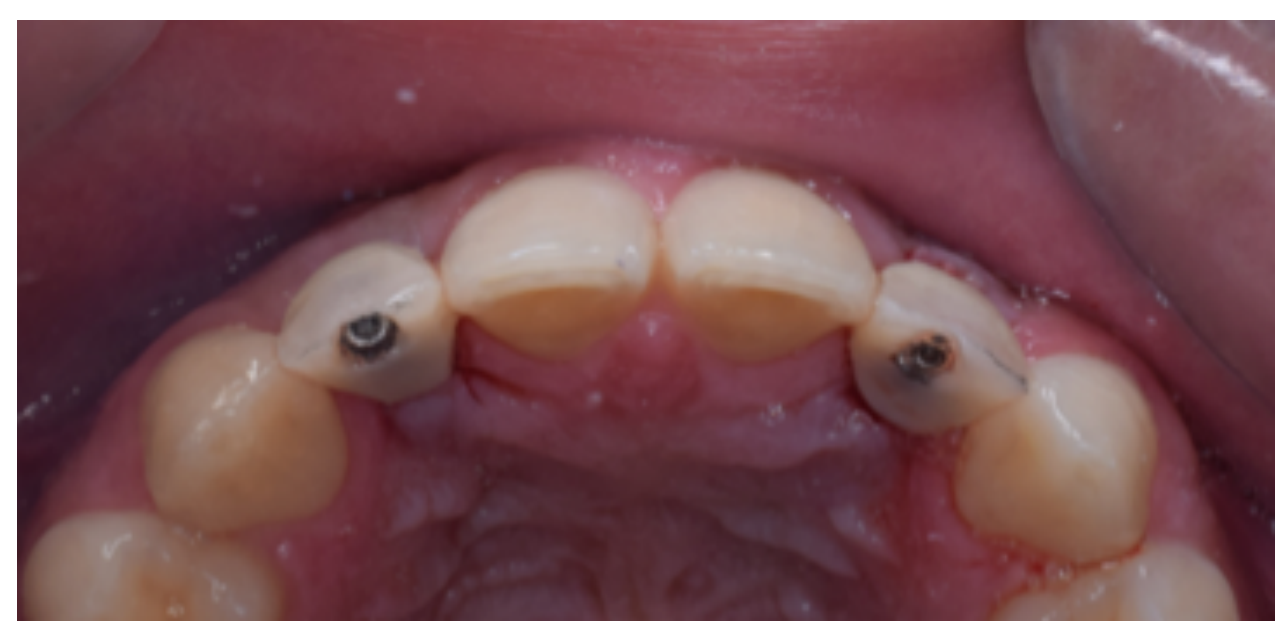

Figura 6. Implante de provisionales. 


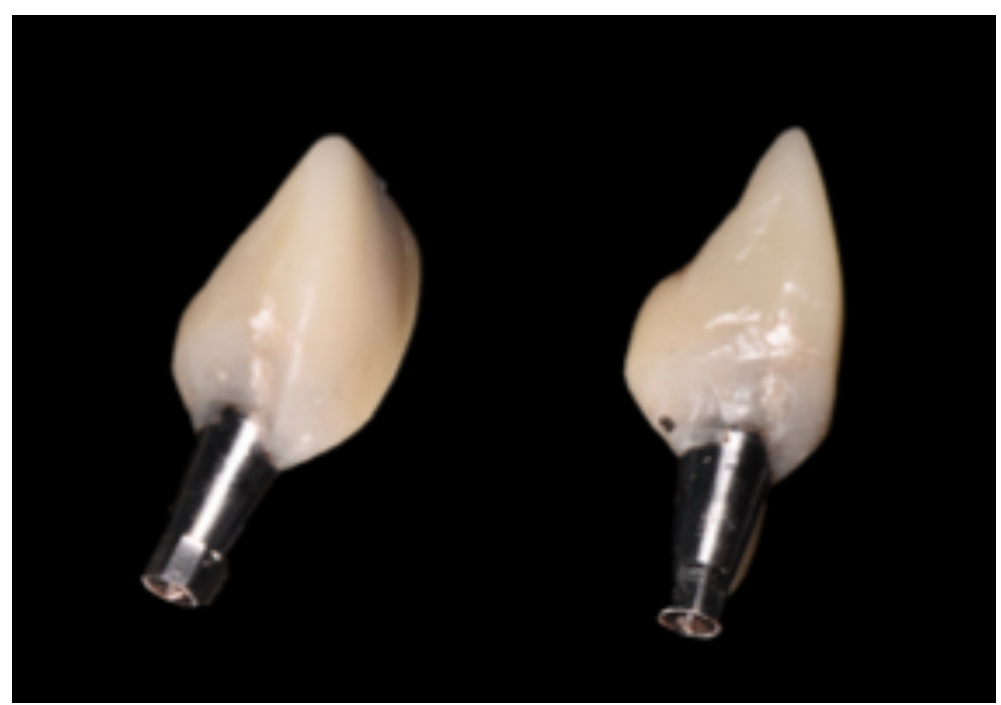

Figura 7. Acrilizado.

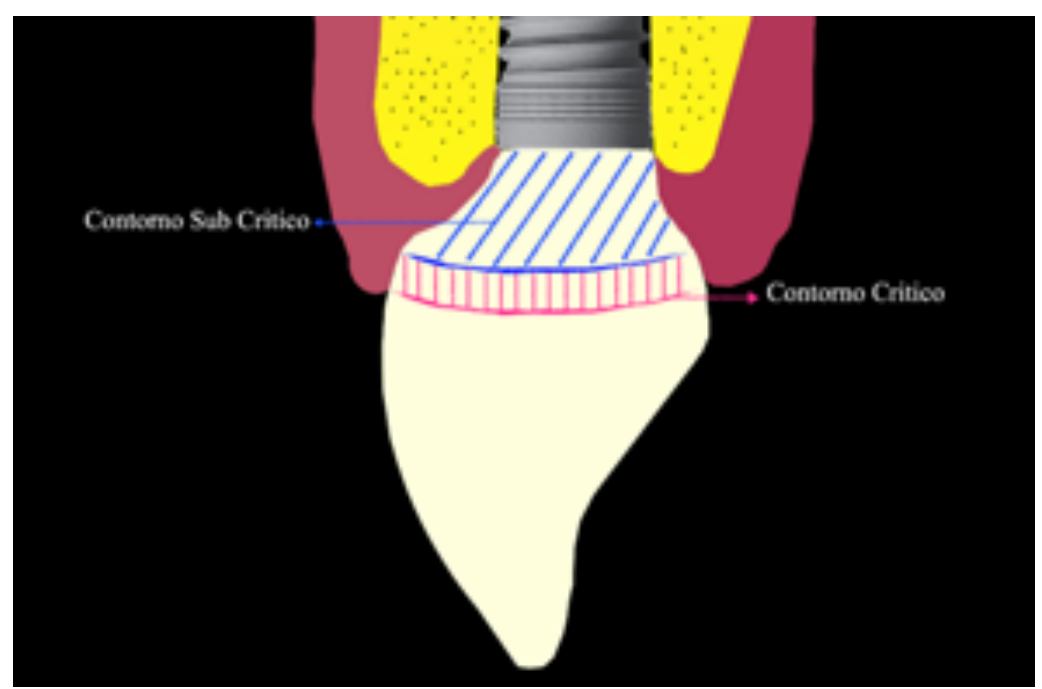

Figura 8. Contorno subcrítico.

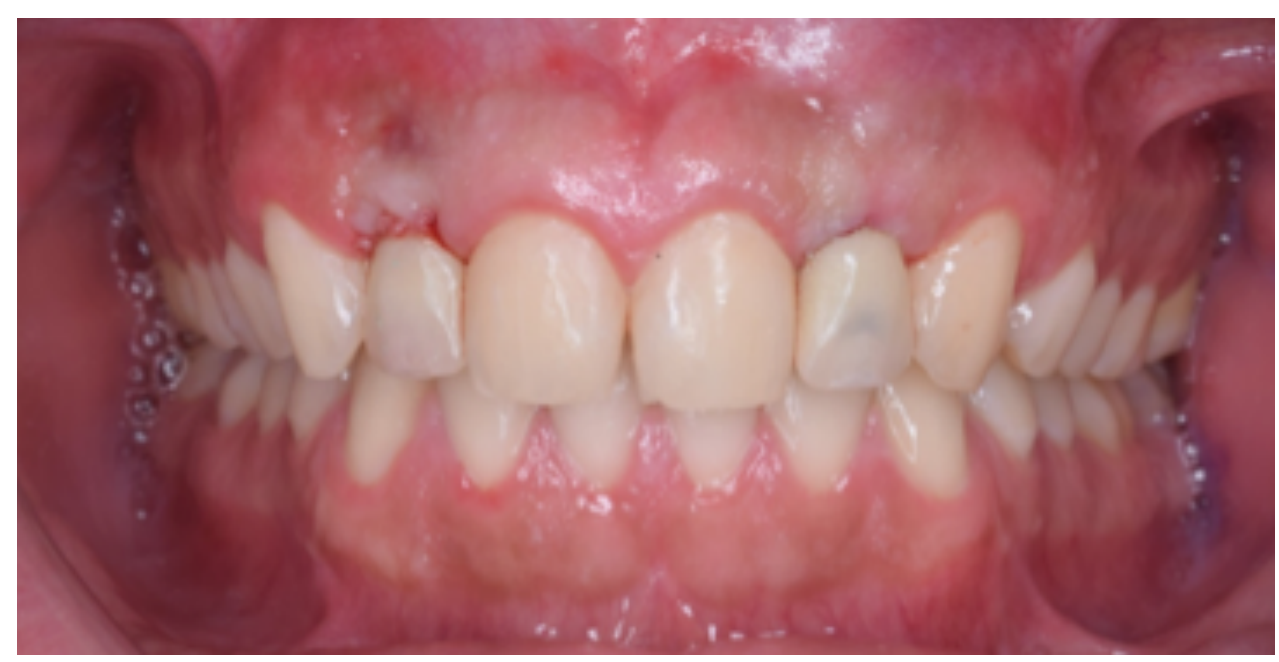

Figura 9. Instalación del provisional a las 2 semanas. 


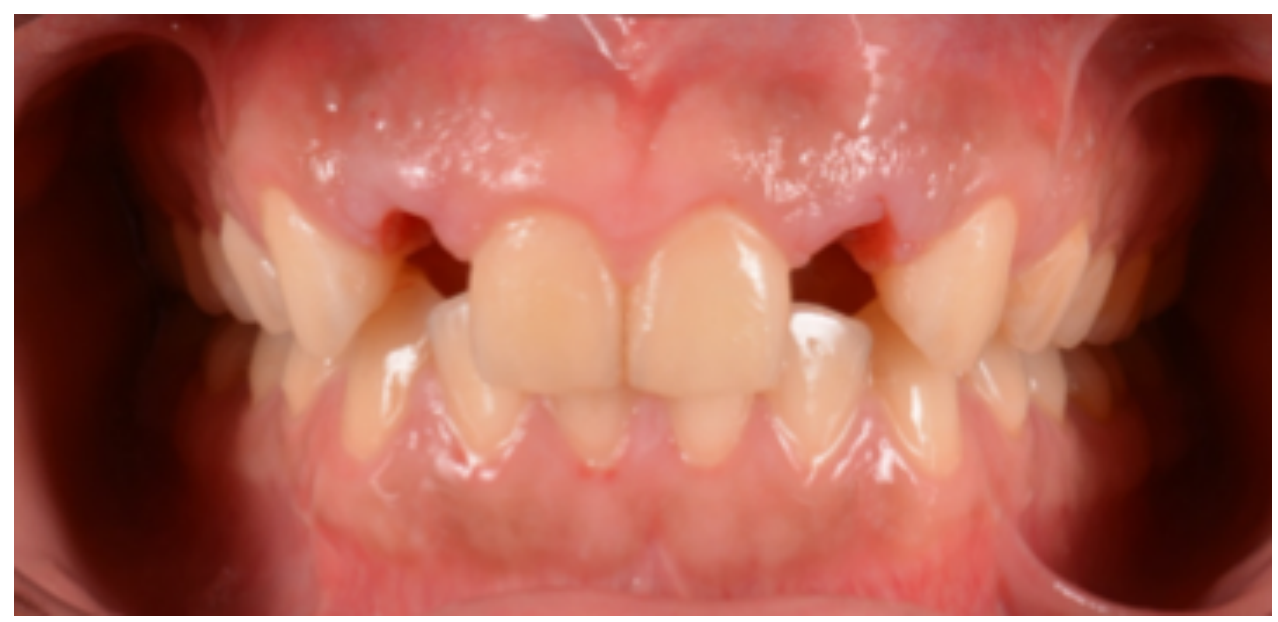

Figura 10. Instalación del provisional a las 4 semanas.

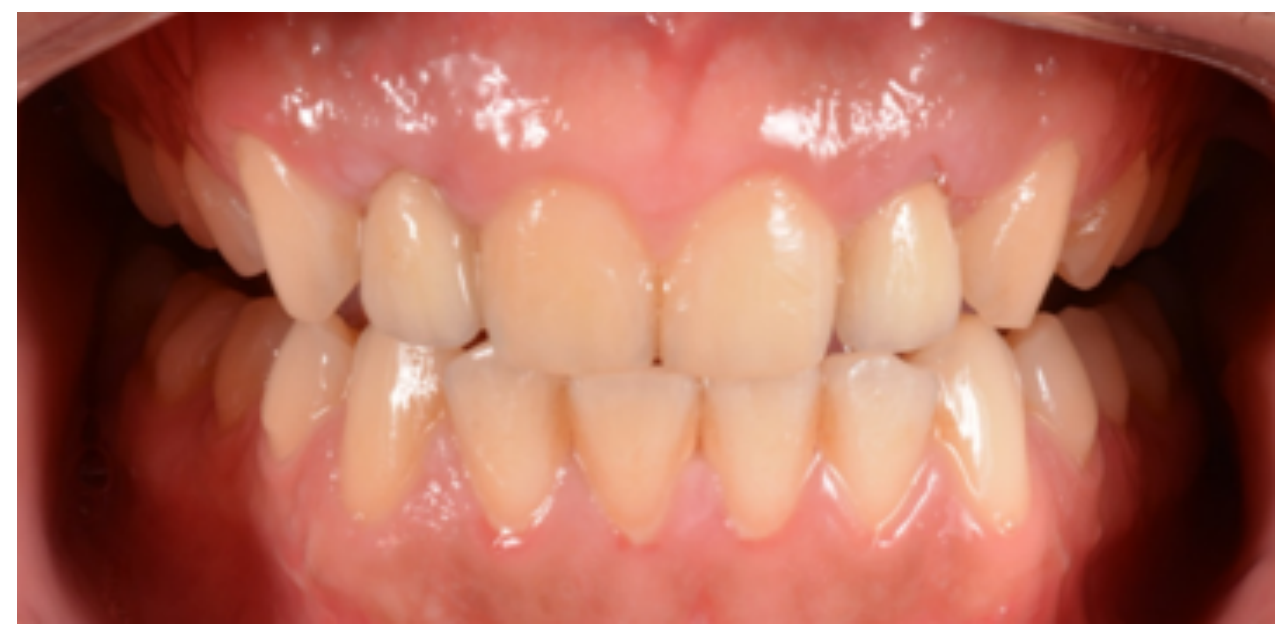

Figura 11. Instalación final con coronas metal cerámicas cementadas.

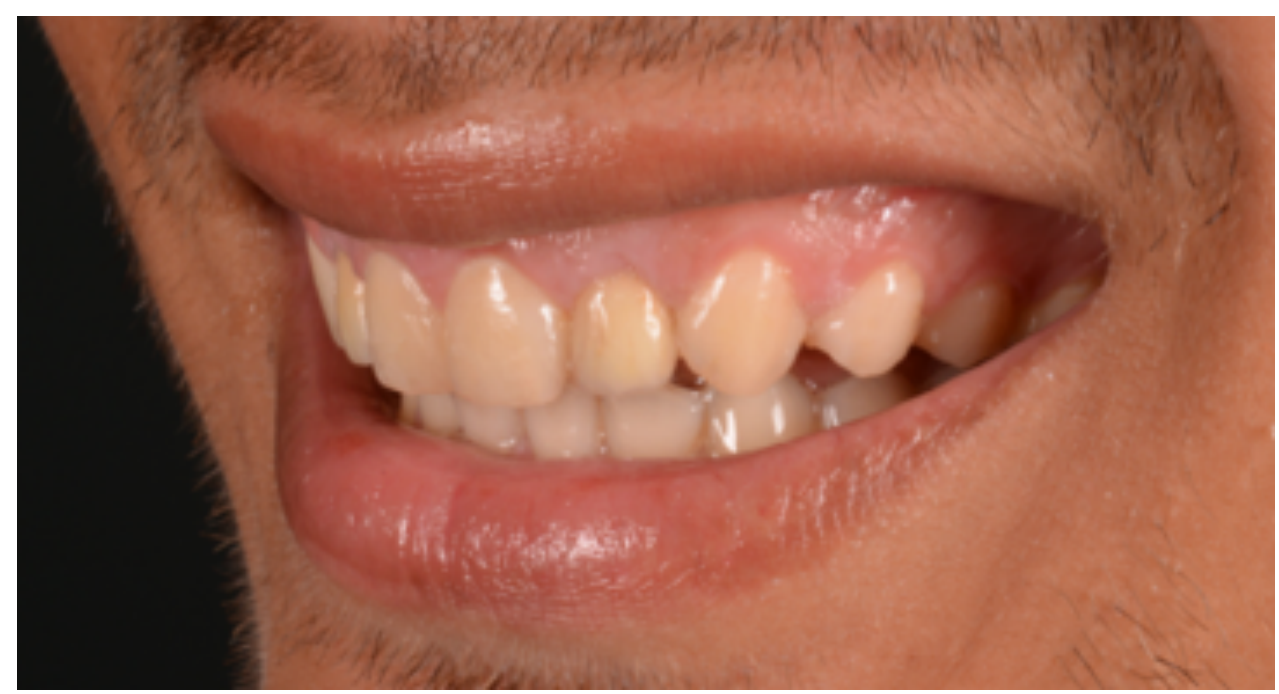

Figura 11. Instalación final con coronas metal cerámicas cementadas. 


\section{DISCUSIÓN}

Un correcto diagnóstico y planificación de un tratamiento es el punto de partida para la restauración de piezas ausentes en el sector anterior (3). Es importante devolver la estética dental como gingival en piezas que involucren la sonrisa, para lo cual es necesario realizar el manejo de los tejidos blandos con provisionales. Se decidió confeccionar provisionales atornilladas ya que las provisionales cementadas presentan un riesgo al generar residuos de cemento lo cual puede ocasionar una periimplantitis (7), otro factor en la selección de provisionales atornilladas es el sellado íntimo que se obtiene con los tejidos blandos provocando un equilibrio biológico, a diferencia de las provisionales cementadas donde los micro-movimientos repetitivos rompen este sellado (8).Clavijo et al., recomiendan realizar la fase de provisionalización con la menor cantidad de citas, modificando los contornos críticos y subcríticos de tal manera que se disminuya el tiempo clínico e incremente la predictibilidad del tratamiento (9). Los protocolos de terapia de implante dental a menudo implican la repetitiva desconexión y reconexión de los cicatrizadores o provisionales para el manejo de los tejidos antes de la instalación definitiva $(10,11)$. Koutouzis et al., mencionan que la desconexión y reconexión del pilar provisional son factores que perturban el contacto íntimo entre el provisional y el tejido conectivo afectando significativamente los niveles óseos marginales periimplantarios $(12,13)$. En el presente caso clínico se manejaron los tejidos blandos con los provisionales sobre implantes en tres citas para minimizar el número de veces de desconexión y reconexión disminuyendo la pérdida ósea marginal. Para el manejo de los tejidos blandos Fürhauser et al., mencionan que existen seis características que se modifican con el contorno crítico y subcrítico estos son: nivel del margen gingival, papila interdental, arquitectura gingival, proceso alveolar, color de la encía y textura gingival (14). Un contorno subcrítico convexo puede mejorar la apariencia de un proceso alveolar vestibular y reducir los efectos de sombra alrededor de la encía vestibular al soportar el tejido gingival. En el presente caso clínico se realizó el manejo de los contornos convexos para soportar un volumen adecuado del tejido gingival. Al momento de realizar el incremento de resina en las restauraciones provisionales para modificar el contorno crítico y subcrítico se debe considerar la reacción de los tejidos periimplantarios, en una primera fase estos se tornarán isquémicos y regresando a un color natural a los 15 minutos, esta reacción debe ser moderada para evitar daños en los tejidos periimplantarios (15).

Conflicto de intereses: los autores no tienen conflicto de interés con este informe.

Financiamiento: Ninguno.

Contribuciones de los autores: todos los autores contribuyeron a este manuscrito.

\section{Correspondencia:}

Raquel Guerra Pérez, Jr. Huiracocha 2331 Dpto 1901. Jesús María, Lima, Perú.

Correo electrónico: raquel.guerra.p@upch.pe

Teléfono :51959266743

\section{REFERENCIAS BIBLIOGRÁFICAS}

1. King P, Maiorana C, Luthardt RG, et al. Clinical and Radiographic Evaluation of a Small-Diameter Dental Implant Used for the Restoration of Patients with Permanent Tooth Agenesis (Hypodontia) in the Maxillary Lateral Incisor and Mandibular Incisor Regions: A 36-Month Follow-Up. Int J Prosthodont. 2016;29(2):147-153.

2. Polder BJ, Van't Hof MA, Van der Linden FP, KuijpersJagtman AM. A meta-analysis of the prevalence of dental agenesis of permanent teeth. Community Dent Oral Epidemiol. 2004;32(3):217-226.

3. Clavijo V, Blasi A. Decision-making process fot restoring single implants. Quintessence of Dental Technology .2017;40:66-88

4. González-Martín O, Lee E, Weisgold A, Veltri M, $\mathrm{Su} \mathrm{H}$. Contour Management of Implant Restorations for Optimal Emergence Profiles: Guidelines for Immediate and Delayed Provisional Restorations. Int J Periodontics Restorative Dent. 2020;40(1):61-70.

5. Su H, Gonzalez-Martin O, Weisgold A, Lee E. Considerations of implant abutment and crown contour: critical contour and subcritical contour. Int $\mathrm{J}$ Periodontics Restorative Dent. 2010;30(4):335-343.

6. Chu SJ, Kan JY, Lee EA, et al. Restorative Emergence Profile for Single-Tooth Implants in Healthy Periodontal Patients: Clinical Guidelines and Decision-Making Strategies. Int J Periodontics Restorative Dent. 2019;40(1):19-29.

7. Chu SJ, Kan JY, Lee EA, et al. Restorative Emergence Profile for Single-Tooth Implants in Healthy Periodontal Patients: Clinical Guidelines and 
Decision-Making Strategies. Int $\mathrm{J}$ Periodontics Restorative Dent. 2019;40(1):19-29.

8. Perrotti V, Zhang D, Liang A, Wong J, Quaranta A. The Effect of One-Abutment at One-Time on Marginal Bone Loss Around Implants Placed in Healed Bone: A Systematic Review of Human Studies. Implant Dent. 2019;28(6):603-612.

9. Clavijo V, Mesquita de Carvalho PF, Soares C. The One-Time Intermediate Abutment-Clinical Application. Quintessence of Dental Technology . 2020;43:7-21.

10. Alves CC, Muñoz F, Cantalapiedra A, Ramos I, Neves M, Blanco J.Marginal bone and soft tissue behavior following platform switch-ing abutment connection/ disconnection: A dog model study. Clin Oral Implants Res. 2015;26:983-991.

11. Becker K, Mihatovic I, Golubovic V, Schwarz F. Impact of abutment material and dis/re-connection on soft and hard tissue changes at implants with platformswitching. J Clin Periodontol 2012;39: 774-780.

12. Koutouzis T, Gholami F, Reynolds J, Lundgren T, Kotsakis GA. Abutment disconnection/reconnection affects peri-implant marginal bone levels: A MetaAnalysis. Int $\mathrm{J}$ Oral Maxillofac Implants. 2017;32(3):575-581.
13. Tallarico M, Caneva M, Meloni SM, Xhanari E, Covani U, Canullo L. Definitive abutments placed at implant insertion and never removed: is it an effective approach? A systematic review and meta-analysis of randomized controlled trials. J Oral Maxillofac Surg. 2018;76(2):316-324.

14. Fürhauser R, Florescu D, Benesch T, Haas R, Mailath $\mathrm{G}$, Watzek G. Evaluation of soft tissue around singletooth implant crowns: The pink esthetic score. Clin Oral Implants Res. 2005;16:639-644.

15. Wittneben JG, Buser D, Belser UC, Brägger U. Periimplant soft tissue conditioning with provisional restorations in the esthetic zone: the dynamic compression technique. Int J Periodontics Restorative Dent. 2013;33(4):447-455.
Recibido : 08-08-2020

Aceptado : 10-06-2021 